\title{
Context Discovery for Model Learning in Partially Observable Environments
}

\author{
Nikolas J. Hemion \\ SoftBank Robotics Europe (Aldebaran) \\ 43 rue du Colonel Pierre Avia, Paris, France \\ Email: nhemion@aldebaran.com
}

\begin{abstract}
The ability to learn a model is essential for the success of autonomous agents. Unfortunately, learning a model is difficult in partially observable environments, where latent environmental factors influence what the agent observes. In the absence of a supervisory training signal, autonomous agents therefore require a mechanism to autonomously discover these environmental factors, or sensorimotor contexts.

This paper presents a method to discover sensorimotor contexts in partially observable environments, by constructing a hierarchical transition model. The method is evaluated in a simulation experiment, in which a robot learns that different rooms are characterized by different objects that are found in them.
\end{abstract}

\section{INTRODUCTION}

This paper presents parts of our ongoing research to develop a method to let robots autonomously learn a model of their sensorimotor interaction with the world. Narrowly defined, model learning can be understood as providing an agent with the means to internally simulate the world, for example to evaluate the outcome of a potential action prior to its execution. This type of model learning is studied in the reinforcement learning literature, where models are typically used to reduce the time that is needed to optimize a policy [1]. Most approaches assume that a suitable state representation of the world is a priori known to the agent, and focus on the problem of letting it estimate state transition probabilities and a reward function through exploration. If however a representation is not provided in advance, this narrow definition has to be extended: when learning a model, an agent not only has to learn the effects of its own actions, it also has to come up with a compact way to represent the state of its environment. Large parts of this representation learning certainly constitute the unsupervised extraction of feature representations from observed data, to reduce the dimensionality of the input and to increase the robustness of the system [2]. But beyond unsupervised feature learning, autonomous agents have to deal with the problem of ambiguity in their observations and unobservable environmental influences on their actions.

This work takes inspiration from theories of perception and cognition in the cognitive science literature [3], [4], to study the problem a naive agent is facing when trying to learn a model while having to deal with ambiguity and latent environmental influences on the outcome of its own actions. The focus here lies on developing a method that allows an agent to construct a hierarchical model of its environment, as a way to discover sensorimotor contexts: situations in which the outcomes of the agent's own actions are predictable. Once the agent is able to recognize such contexts, it can learn to predict the effect of its own actions for each of the contexts (the demonstration of which however lies out of the scope of this paper).

\section{DISCOVERING SENSORIMOTOR CONTEXTS TO MINIMIZE PREDICTION ERROR}

Central to our approach, and as argued in more detail elsewhere [5], is the idea that an agent should try to discover predictable patterns of interaction in the flux of sensorimotor observations. In the following, the argument will be briefly summarized.

In line with so-called "predictive processing" theories of cognition, which have increasingly received attention in the recent cognitive science literature [3], [6], [7], we argue that autonomous agents need to learn to predict immediate and future sensorimotor observations, to be able to react adaptively. A central factor driving the learning and exploration of an agent should therefore be the goal to minimize its error of prediction. To formalize this, consider an agent interacting with its environment. We can describe the stream of its sensorimotor observations as the transition probability

$$
\operatorname{Pr}\left(\mathbf{x}^{\prime} \mid \mathbf{x}, \pi, \mathbf{e}\right)
$$

expressing the probability for the agent to observe $\mathrm{x}^{\prime}$ after having observed $\mathbf{x}$, while executing the control policy $\pi$. Additionally, we let the probability distribution depend on a latent variable e, which represents the current "agent-environment configuration": it summarizes all external factors that influence the outcome of the agent's actions. For example, imagine a robot with a control policy to grasp a bottle: executing this policy will obviously only have a chance of success if there is a bottle in reach of the robot in the current situation. As another example, imagine two identical robots, standing in a corridor in front of two identical looking doors, one leading to a kitchen, the other leading to a dining room. The sequence of observations that the two robots would make when opening and passing through the respective doors would of course be very different (one would probably see a fridge, while the other would probably see a dining table). This environmental influence, or sensorimotor context, is summarized in an abstract way by the variable e. 
In practice, it is usually the designer of the robot who ensures that the robot only executes its actions in suitable contexts. Either this is the case because the robot never leaves a well-defined environment (such as a laboratory), or the robot is manually provided with a detection mechanism (for example, a "bottle detector", in the case of the first example above). In both cases, the model learning is implicitly turned into a supervised learning problem, as the human expert determines a suitable context $\mathbf{e}$ for a task at hand and designs the robot and/or its environment in such a way as to ensure that the context remains suitable throughout every task execution. However, when the aim is to build truly autonomous robots, this becomes unfeasible. Instead, we require a procedure to let robots learn in an unsupervised manner (or only depend on supervision through natural interaction, to the extent that it is also received by infants from their caregivers).

Thus, the agent cannot estimate the above transition probability when it does not have access to a supervisory signal. What the agent does observe as it tries to execute a policy $\pi$ in a number of unobservable sensorimotor contexts $\mathbf{e}_{i}, i \in$ $\{1,2, \ldots, N\}$ is a marginalized version of the probability distribution

$$
\widehat{\operatorname{Pr}}\left(\mathbf{x}^{\prime} \mid \mathbf{x}, \pi\right)=\sum_{i=1}^{N} \operatorname{Pr}\left(\mathbf{x}^{\prime} \mid \mathbf{x}, \pi, \mathbf{e}_{i}\right),
$$

which summarizes the probability of observing $\mathbf{x}^{\prime}$ after $\mathbf{x}$ while executing the policy $\pi$ across sensorimotor contexts.

Naturally, the entropy of this marginalized distribution increases with the number of sensorimotor contexts which influence the outcome of the agent's executed actions. This in turn means that the agent's prediction error rate would increase, were it to make predictions of future observations solely based on an estimate of this distribution. By implication, this means that the agent can minimize its prediction error by demarginalizing the observed transition probability distribution. The entropy would be minimal were the agent to achieve a distribution that corresponded to the actual values of the latent variable e: as this variable represents all external factors that influence the outcome of the agent's actions, any remaining stochasticity would be intrinsic to the agent's own policy.

One might be inclined to think of the variable $\mathbf{e}$ as the set of all possible states of the entire universe. But this would of course be entirely misleading: instead, it is helpful to conceive of it as the most compact way to encode all qualitatively different situations the agent can face, given its sensorimotor apparatus. This is related to the concept of "sensorimotor contingency" from the theory of perception proposed by O'Regan and Noë [4].

Assuming that an agent has successfully demarginalized the transition probability distribution by constructing an internal model, it can estimate the current state e by tracking the likelihood of individual hypotheses over time: making predictions about future observations when assuming certain states, and comparing the predictions with actual observations, to update likelihoods correspondingly. Importantly, this implies that the distinguishing property on which the agent relies to decide whether two observations belong to the same sensorimotor context or not is temporal adjacency: given a high likelihood of a certain context $\mathbf{e}$, the agent assumes that the next observation will also correspond to the same context. Furthermore, the agent can test whether two observations are part of the same sensorimotor context by trying to produce the one observation after having seen the other, by means of its own actions. If the agent can reliably transition between two observations whenever it believes that a certain sensorimotor context has a high likelihood, the agent can safely assume that both observations are part of the same sensorimotor context and can update its model accordingly. On the contrary, if the agent cannot produce some observation, it can be inferred that this observation does not belong to the current sensorimotor context.

To summarize, we can say that observations that belong to the same sensorimotor context share the property that the agent can transition between them using its own actions, or in other words, they are "reachable" from one another for the agent. In contrast, observations that are not reachable in this sense do not belong to the same sensorimotor context. The task to discover sensorimotor contexts within the flux of sensorimotor observations can thus be reformulated as one of finding sets of observations, for which the agent observes a high probability of transitioning within the set, but observing transitions out of the set only with a low probability.

\section{A. Mathematical Formalization}

We can formalize this by calling $T_{\pi}$ the transition probability matrix corresponding to the probability distribution defined in Eq. 2, which when interpreted as an adjacency matrix defines a graph of observations, with edges representing the probability of transitioning between observations when the agent is executing the policy $\pi$. The task of finding sensorimotor contexts is thus related to the problem of finding clusters of densely connected nodes in this graph. This can be approximately achieved through spectral clustering [8], using the method suggested by $\mathrm{Ng}$ and colleagues [9], in the following way.

We first solve the eigenvalue problem for the transition probability matrix $T_{\pi}$ to find the $k$ largest eigenvalues $\lambda_{1}, \lambda_{2}, \ldots, \lambda_{k}$ and associated eigenvectors $u_{1}, u_{2}, \ldots, u_{k}$, and form the matrix

$$
U=\left[\begin{array}{llll}
u_{1} & u_{2} & \ldots & u_{k}
\end{array}\right] \in \mathbb{R}^{m \times k}
$$

where $m$, is the number of discrete sensorimotor states that the agent can observe. The matrix $U$ is then normalized such that each row has unit length, resulting in the matrix $V$ with entries

$$
V_{i, j}=\frac{U_{i, j}}{\sqrt{\sum_{l} U_{i, l}^{2}}} .
$$

Since $V$ is computed from the observation transition probability matrix $T_{\pi}$, each row still corresponds to one observation 


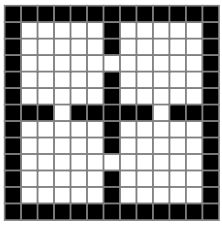

room world

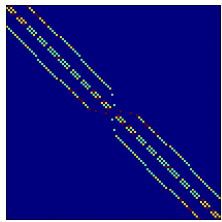

state transition probability matrix

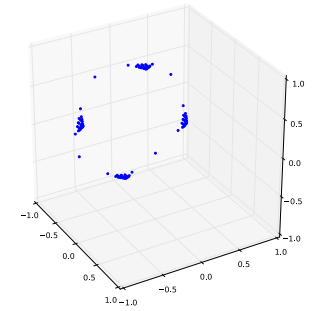

states mapped into spectral space (3-dimensional projection)
Fig. 1. The result of mapping the set of states from the room environment shown on the left into a 4-dimensional spectral space (projected into three dimensions for visualization), based on the observed state transition probabilities (as shown by their transition probability matrix). See text for more details.

x. By treating each row in $V$ as a vector in $\mathbb{R}^{k}$, we can thus map the observations into a $k$-dimensional "spectral space". As we will see below, this mapping has the property that two observations sharing strong transition probabilities tend to lie close together, whereas observations with low transition probabilities between them lie further apart. This property can be exploited to discover sets of observations that tend to co-occur by clustering the points in this spectral space, for example using K-means.

The idea to use graph clustering methods to partition a state graph has already been suggested in the reinforcement learning literature [10], [11], but with a different motivation: the aim of these works is to discover "subgoals", to speed up learning convergence in reinforcement learning (see also [12]). The idea is that "bottlenecks" (such as doorways in a navigation task) are important subgoals when discovering a policy, and they can be characterized as state transitions with low probability between two clusters of densely connected states. In contrast, here we are interested in discovering densely connected clusters, with the aim to demarginalize the transition probability and to discover sensorimotor contexts.

\section{B. Example: Four room world, fully observable case}

To exemplify the mapping of observations into the spectral space, consider the "room world" depicted in Figure 1, in which an agent is placed that is able to move up, down, left, and right. In this example, we consider the fully observable case, meaning that the agent makes a unique observation in each position, allowing the agent to unambiguously recognize whenever it reaches the same position. Environments similar to this one are often studied in the reinforcement learning literature.

As the agent explores its environment by executing an exploratory policy $\pi$ (for example a random walk), it can record the transition probabilities between observations in a transition probability matrix $T_{\pi}$ to estimate the marginal probability distribution in Equation 2. The resulting transition probability matrix for the policy in which each of the agent's four actions have equal probability to be selected is shown in Figure 1 .

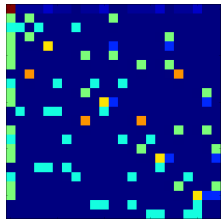

observation transition probability matrix

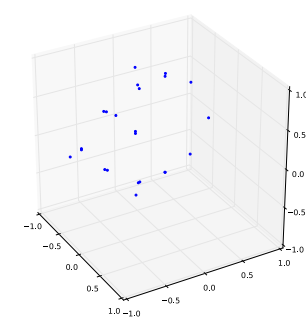

observations mapped into spectral space (3-dimensional projection)
Fig. 2. The result of mapping the set of observations from the room environment shown in Figure 1 into a 4-dimensional spectral space (projected into three dimensions for visualization), based on the observed observation transition probabilities (as shown by their transition probability matrix). See text for more details.

If we map the 104 unique observations (corresponding to the 104 unique positions that the agent can be in) into a 4-dimensional spectral space using the method described above, we obtain a distribution of observations as shown in the figure (projected into three dimensions for visualization). We clearly see four dense clusters, each composed of all the observations belonging to one room. In between the clusters lie the four "doorway" states, through which the agent passes when moving from one room into another.

\section{Example: Four room world, partially observable case}

Now consider the same case in which the agent is not able to directly observe its position in the world. Instead, we equip it with a sensor to see only the cells surrounding its current position. This agent will not be able to distinguish between any positions in which it makes the same observation, for example the upper left corners of all four rooms seem identical to it. However, to be able to predict the outcome of its own actions, it needs to know in which room it is located: moving down from the upper left corner of the bottom right room results in seeing the room's left door, whereas moving down from the upper left corner of the upper left room results in seeing a wall.

Observations are ambiguous in this partially observable case. The agent's actions can lead to different transitions between observations, depending on the current sensorimotor context (here: which room the agent currently is in). This ambiguity also manifests itself in the mapping into the spectral space of the observation transition probability matrix: while some of the observations lie closer together than others (because for example corners tend to transition to walls, but never to other corners), the fact that the world consists of four separate rooms is not recognizable based on the distribution in this space (in contrast to the fully observable case).

\section{Dealing with partial observability by means of hierarchy}

To overcome the difficulty of partial observability, we make use of the following intuition: while a single observation can be ambiguous, there will always be a sequence of observations that will unambiguously identify any event, given the sequence is sufficiently long. For example, a single frame from a 

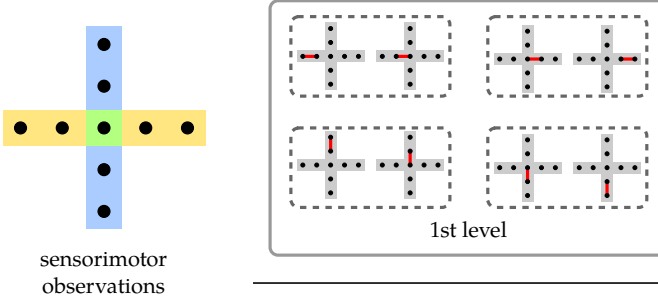

observations

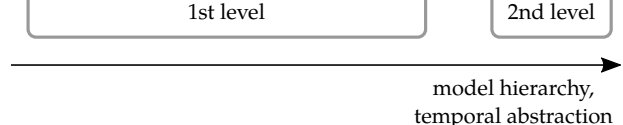

Fig. 3. How a model composed of a hierarchy of observation transitions extends the temporal horizon across levels and provides an agent with a form of memory (see text for details).

video of a ball rolling along a plane would not allow a viewer to determine in which direction the ball is rolling. Two consecutive frames from the same video however are sufficient to determine the direction of the ball's movement.

What sequence length is necessary to disambiguate observations differs of course from situation to situation - it is not possible to fix it a priori. Instead, the agent should be able to flexibly extend the sequence length if necessary (i.e. when it is facing ambiguous observations). We can achieve such a behavior by constructing a model which consists of a hierarchy of transitions: in such a model, each successive level increases the temporal horizon of representation. New representational levels can be constructed by first grouping together "similar" transitions on the respective lower level (ones that frequently co-occur, determined using the spectral method described above), and subsequently determining which transitions between these groups of transitions are observed.

To make this idea more clear, consider the example shown in Figure 3. On the left of the figure, an abstract example of two sensorimotor contexts is depicted, one shown in yellow, the other shown in blue. Each context consists of five observations (shown as black dots), most of which unambiguously belong to only one of the two contexts. Only the observation in the center, at the "intersection" of the two contexts (shown in green), can belong to either the one or the other context. Whenever the agent makes this observation, it thus cannot determine based on the observation alone what the current context is. In this example, a single transition is sufficient to disambiguate contexts - if the agent transitions from the central observation "vertically", it currently is in the blue context; otherwise, if it transitions "horizontally", it is in the yellow context. The first level of the model (shown on the right of the figure) represents transitions between observations. The agent can further create a second layer to discover the actual latent structure of the environment, by first grouping together "similar" transitions (ones that frequently co-occur; shown as dashed boxes), and subsequently determining which transitions between these groups of transitions it can observe. The second level thus represents higher-order transitions, each one corresponding to four simple transitions.

Indeed, as illustrated by this example, the construction of such a hierarchical model can be used to demarginalize the
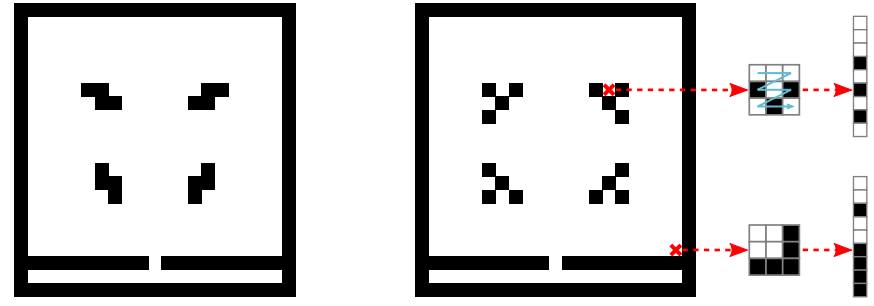

Fig. 4. Examples for the two types of rooms that were generated in the simulation experiment: an $s$-room on the left, and a $y$-room on the right. The room types differ in that they each have a unique set of "objects" placed inside them. A simulated robot navigates the room environment my moving up, down, to the left, or to the right. It observes only the fields immediately neighboring its current position, as a binary vector with nine entries, as shown on the right.

transition probability distribution: by constructing an internal hierarchical model, the agent can estimate the latent structure of the environment and obtain an internal representation of sensorimotor contexts e. In the next section, this will be demonstrated by means of a simulation experiment.

\section{Simulation EXPERIMENT}

\section{A. The simulated world}

The simulation experiment should emulate the experience of a robot that explores different rooms, without having any prior knowledge whatsoever about the nature of rooms (neither that there exist different kinds of rooms, nor that rooms consist of walls, that there are objects inside rooms, etc.). We define two room types: $y$-rooms and $s$-rooms, each characterized by having a unique set of "objects" that are placed inside them. A simulated robot is placed in the room environment, and can navigate along a discrete grid of positions, with the four primitive actions of moving up, down, to the left, and to the right. At each position, the robot observes its environment in the form of a binary occupancy vector of length 9, measuring only the grid positions that are immediately surrounding its current position. Figure 4 shows a visualization of the simulation.

The robot explores its environment using a simple policy: at each time step, it selects any of its valid actions (ones leading to an unoccupied grid position) with equal probability. To let the robot experience different instances of each room, a randomization mechanism is implemented in the simulation, in the following way. Each room has a door in its south wall, leading to a corridor. Whenever the robot leaves a room through this door, a new room is randomly generated, either an $s$-room or a $y$-room with equal probability. Rooms have a size of $50 \times 50$ tiles including walls, and 20 objects from the room's set are randomly placed in the room, ensuring that they are not overlapping or directly adjacent to one another or to a wall.

\section{B. Simulation results}

The robot explores its environment for a total of $10,000,000$ time steps. In its environment, the robot encounters 46 unique 

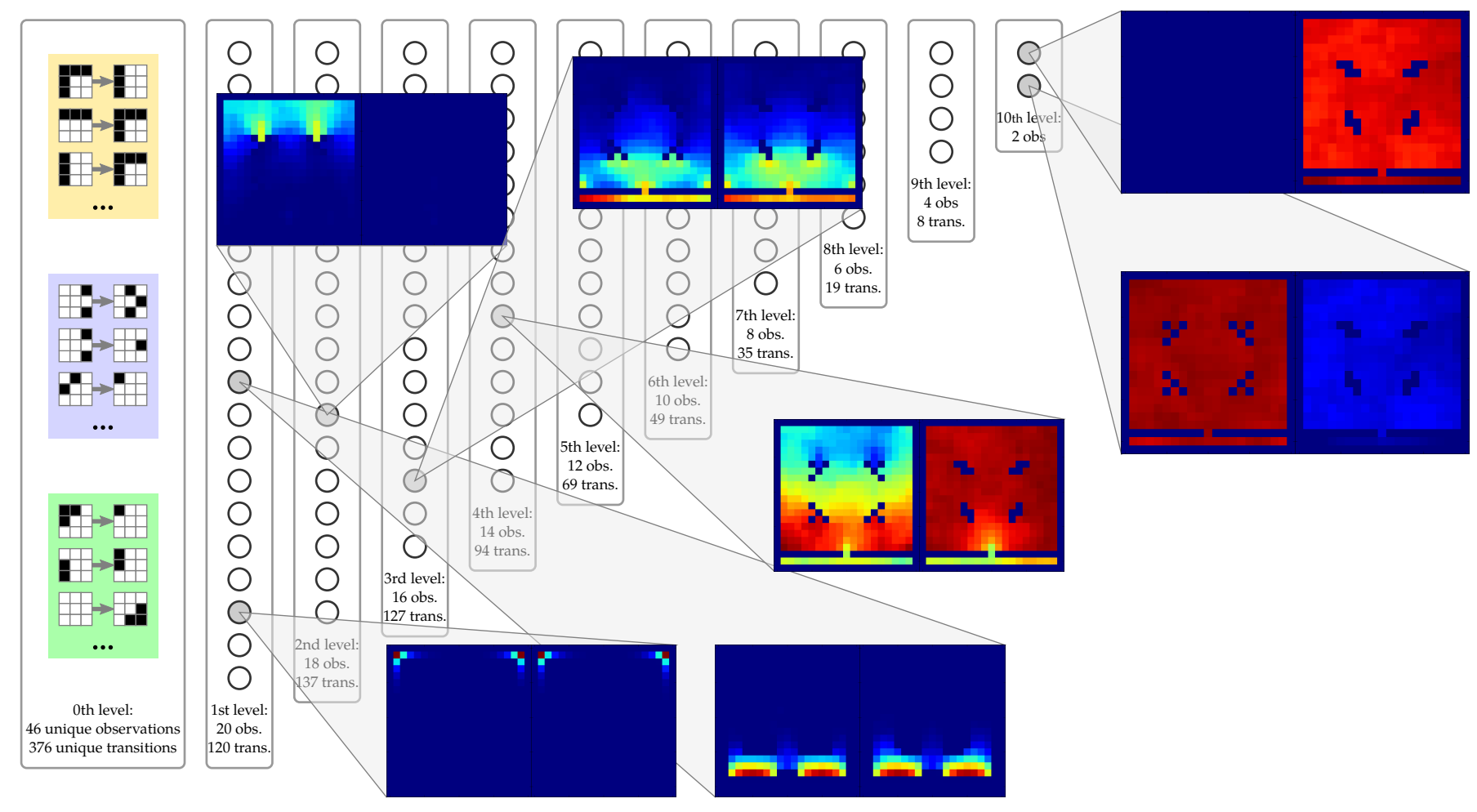

Fig. 5. Summary of the results of the simulation experiment. On the left (0th level), simple transitions between observations are shown. Some of these transitions are between room features (marked in yellow, for example moving from a corner to a wall). Others are specific to one of the objects ( $y$-type objects marked in blue, $s$-type objects marked in green). The result of applying the method for constructing a hierarchical transition model is shown by visualizing for some nodes, where in the test rooms the agent observes the encoded transitions. The higher the hierarchical level, the larger the temporal abstraction of the encoded transitions is. Intermediary nodes encode experiences such as "entering a room from the corridor", while the two nodes on the highest hierarchical level encode the experiences "being in a $y$-type room" and "being in an $s$-type room", respectively.

observations, some of which correspond to room features (walls and corners), while others correspond to object parts.

After the robot has completed its exploration, a hierarchical transition model is constructed using the learning method described above, in the following way (see also Figure 5. First, each unique transition between observations (376 in total) is represented on the "0th level", and a $376 \times 376$ transition probability matrix is constructed based on the observation sequence. The mapping into its 3-dimensional spectral space is computed, and spectral clustering in this space is performed using agglomerative clustering, to find 20 clusters of transitions. Each of the clusters is then treated as a "metaobservation" for the construction of the next hierarchical level.

This procedure (finding all unique transitions between observations on the respective lower level, constructing a transition probability matrix, performing spectral clustering) is then repeated to form a total of 10 hierarchical levels, where the number of clusters is decreased by 2 on each consecutive level, giving $18,16, \ldots, 4,2$ clusters, respectively.

To visualize the outcome of the training, two "test rooms" (one for each room type) are created (the ones shown in Figure 4). The robot is then allowed to explore each of the two test rooms for 500,000 time steps (a duration long enough to achieve a close to uniform distribution over the positions of the robot). A "heat-map" is used to visualize what experiences the units across the representational hierarchy come to encode: it shows the probability of the robot being in a certain position whenever a transition sequence is observed that is encoded by a given unit, up until the observation of the next transition sequence.

As seen in the Figure 5, lower levels of the representational hierarchy encode experiences that are of short duration and specific, whereas higher levels encode experiences that have longer durations and become more abstract. In particular, on the first levels of the hierarchy, experiences of the robot as running into a corner or moving along a wall are represented. Also experiences that are specific to objects are encoded by some units, as for example seen in the case of a unit on the second level, which represents the experience of approaching two of the $y$-type objects from the top. On the third level, we already find units representing more abstract experiences, such as "entering a room from the corridor". One of the units on the fourth level seems to have already captured the experience of roaming the $s$-type room, but is not yet entirely selective: the transition sequences that it represents are also observed in the lower part of the $y$-type test room. On the last hierarchical level, the two units have finally separated the experience of being in either one of the two room types. 


\section{DISCUSSION}

The simulation experiment shows that the agent can discover sensorimotor contexts: by exploring its environment, it learns to distinguish the two different room types. For the agent's policy, the observed transition probabilities of course depend on the room in which it currently is (for example, it will never move from a wall to a $y$-type object in an $s$ type room). Therefore, the agent would not have been able to learn a good predictive model directly from the sequence of observations that it made during its exploration. Given the learned hierarchical transition model however, it could now separate experiences belonging to either one of the two room types. This way, the agent has constructed an internal representation of sensorimotor contexts, which it can use to demarginalize the observed transition probability, and thus can reduce its prediction error when learning a predictive model.

The work presented in this paper still leaves a number of open questions unaddressed. For example, in the simulation experiment, the number of clusters for each hierarchical level was manually selected. It would be desirable to find a method to automatically determine a good number of clusters for the construction of each new level, for example by using an Entropy-based measure. Furthermore, from an external point of view it makes sense to distinguish two contexts in the simulated environment: one for each room type. Consequently, having a representational hierarchy with two states at the top level is a suitable choice. Generally however, it seems like a mechanism is required to determine when a good demarginalization has been already achieved, such that no further hierarchical levels might be needed to represent the environment.

In the simulation experiment described in this paper, the agent's observation and action spaces were discrete. It should however be noted that the presented method can also deal with continuous inputs, by introducing a first step of discretization. For example, observations lying in a continuous space could be discretized using K-means (cf. [5]).

In future work, the presented learning method for hierarchical transition models will be combined with the training of predictive models. In such a setting, the method presented here can be used to discover sensorimotor contexts, such that subsequently a predictive model can be learned for each individual sensorimotor context. This way, an agent could learn predictive models for its own actions also in partially observable environments, where latent environmental factors influence the outcome of the agent's actions.

\section{REFERENCES}

[1] T. Hester and P. Stone, "Learning and using models," in Reinforcement Learning. Springer, 2012, pp. 111-141.

[2] Y. Bengio, A. Courville, and P. Vincent, "Representation learning: A review and new perspectives," Pattern Analysis and Machine Intelligence, IEEE Transactions on, vol. 35, no. 8, pp. 1798-1828, 2013.

[3] K. Friston, "The free-energy principle: a unified brain theory?" Nature Reviews Neuroscience, vol. 11, no. 2, pp. 127-138, 2010

[4] J. K. O'Regan and A. Noë, "A sensorimotor account of vision and visual consciousness," Behavioral and brain sciences, vol. 24, no. 05, pp. 939973, 2001.

[5] A. Laflaquière, N. Hemion, M. Garcia Ortiz, and J.-C. Baillie, "Grounding perception: A developmental approach to sensorimotor contingencies," in IEEE/RSJ International Conference on Intelligent Robots and Systems (IROS). Hamburg, Germany, 2015.

[6] A. K. Seth, "A predictive processing theory of sensorimotor contingencies: explaining the puzzle of perceptual presence and its absence in synesthesia," Cognitive neuroscience, vol. 5, no. 2, pp. 97-118, 2014.

[7] J. Hohwy, The predictive mind. OUP Oxford, 2013.

[8] U. Von Luxburg, "A tutorial on spectral clustering," Statistics and computing, vol. 17, no. 4, pp. 395-416, 2007.

[9] A. Y. Ng, M. I. Jordan, Y. Weiss et al., "On spectral clustering: Analysis and an algorithm," Advances in neural information processing systems, vol. 2, pp. 849-856, 2002.

[10] Ö. Şimşek, A. P. Wolfe, and A. G. Barto, "Identifying useful subgoals in reinforcement learning by local graph partitioning," in Proceedings of the 22nd international conference on Machine learning. ACM, 2005, pp. 816-823.

[11] S. Mannor, I. Menache, A. Hoze, and U. Klein, "Dynamic abstraction in reinforcement learning via clustering," in Proceedings of the twenty-first international conference on Machine learning. ACM, 2004, p. 71.

[12] A. G. Barto and S. Mahadevan, "Recent advances in hierarchical reinforcement learning," Discrete Event Dynamic Systems, vol. 13, no. 4, pp. 341-379, 2003. 